\title{
INTEGRACIÓN REGIONAL Y DESARROLLO: EXPERIENCIAS DE DESARROLLO LOCAL EN MERCOSUR
}

\author{
REgIONAL INTEGRATION AND DEVELOPMENT: EXPERIENCES OF LOCAL \\ DEVELOPMENT IN MERCOSUR
}

Isabel Clemente Batalla

\begin{abstract}
RESUMEN
La asociación de integración regional y desarrollo como fundamento para la organización de los bloques de integración en América Latina ha sido una línea constante y recurrente en las teorías latinoamericanas sobre relaciones internacionales y política exterior, aun cuando los modelos implementados históricamente demuestran aproximaciones diferentes y a veces también contradictorias en cuanto a los objetivos, los métodos y los agentes del desarrollo. Una orientación sostenida en Mercosur desde inicios del tercer mileno propone el desarrollo local como modelo que incluye la dimensión territorial y los objetivos de desarrollo social, en un proceso favorecido por los avances hacia la descentralización, el empoderamiento de las comunidades locales y la creación de nuevos vínculos entre diferentes escalas espaciales: global, regional, nacional y local sub-nacional. El artículo examina los alcances de las experiencias del modelo de desarrollo local en el proceso de integración de MERCOSUR, en sus dimensiones territorial, económico-social y cultural y el papel cumplido por la iniciativa local. Se adopta el concepto de "local" no meramente como una réplica en menor escala espacial de determinantes de naturaleza sistémica, sino como una realidad dotada de aspectos específicos e identidades propias, valores compartidos, capacidad de iniciativa y normas internalizadas.
\end{abstract}

\section{PALABRAS CLAVE}

Desarrollo - MERCOSUR - Sociedad civil - Instituciones locales - Territorio

\begin{abstract}
The association of regional integration and development as the foundation for the organization of integration groups in Latin America has been a constant and recurrent direction in Latin American theory of international relations and foreign policy, even if the models actually implemented reveal different approaches and sometimes even contradictory as to the goals, methods, and agents of development. A line of argument held in Mercosur since the beginning of the third millennium puts forward local development as a model that includes the territorial dimension, and the goals of social development in a process moved by the advancement towards decentralization, empowerment of local communities, and the creation of new links among diverse spatial scales: global, regional, national, and local sub-national. The article examines the scope of the experiences of the model of local development in Mercosur process of integration, in its territorial, socio-economic, and cultural dimensions, and the role played by local initiative. It adopts the concept of "local" not just as a copy in reduced scale of determinants of systemic nature, but as a reality having specific aspects and its own identity, shared values, initiative capacity, and internalized norms.
\end{abstract}

\section{KEY WORDS}

Development - Mercosur - Civil Society - Local institutions - Territory 


\section{INTRODUCCIÓN}

Los orígenes de la integración regional en América Latina han estado asociados a proyectos de desarrollo económico desde que Raúl Prebisch presentara su propuesta en la CEPAL, luego implementada en la creación del primer bloque subregional, el Mercado Común Centroamericano. Esta asociación ha experimentado grandes cambios desde entonces en el marco del proceso de profundas transformaciones en la economía mundial, en el abordaje del desarrollo económico y en los modelos de integración regional, aun cuando el principio originario se ha mantenido.

Por otra parte, la diversidad en modelos de integración regional y de desarrollo y el cambio que se registra como resultado de la evolución de los conceptos y las estrategias, complejiza el análisis de la relación entre ambos procesos. Un problema de metodología es la definición de indicadores de desarrollo comparables, sobre la cual existen propuestas divergentes: Lugo (2007) propone el empleo por considerar que este es la fuente principal de ingresos de la mayoría de las familias mientras que Ibrahim y Alkire (2007) siguiendo el análisis de Amartya Sen, optan por capacidad de iniciativa y empoderamiento.

En América Latina, los intentos de construcción de indicadores de desarrollo marcan un avance en la investigación teórica pero difieren sustantivamente en su aplicación en investigación empírica. Los debates sobre el desarrollo y la integración regional se han asociado con la diversidad de opciones: entre el libre comercio y el proteccionismo, el regionalismo abierto y el regionalismo entendido como estrategia para la diversificación productiva. La renovación en las teorías del desarrollo se origina en la incorporación de nuevas dimensiones inicialmente no contempladas: la inclusión social, la formulación de políticas públicas, la cultura, el medio ambiente y la sustentabilidad. La dimensión territorial, central en los procesos de integración regional, se constituye también en un eje importante en propuestas de desarrollo que incorporan la escala espacial como factor determinante con diferentes alcances: global, regional, local, sub-nacional. La política de descentralización con transferencia de competencias de gobierno a entidades locales fortalece la adscripción al territorio de los vínculos sociales, productivos y de infraestructura. Nuevas alternativas como desarrollo endógeno, desarrollo local y desarrollo sostenible ingresan en la discusión sobre el modelo de integración apropiado para alcanzar los objetivos del desarrollo. 
La primera sección de este artículo discute las diversas contribuciones teóricas sobre la relación entre propuestas de desarrollo y modelos de regionalismo e integración de América Latina. La segunda sección se propone analizar las alternativas de desarrollo local y desarrollo sostenible y sus respectivas aproximaciones al territorio. La tercera sección considerará dos áreas territoriales del MERCOSUR donde se han puesto en práctica políticas de desarrollo con diversas escalas territoriales: los proyectos de la nueva agenda binacional de desarrollo e integración trans-fronteriza de Brasil y Uruguay y el eje de comunicaciones articulado en torno al puerto de Nueva Palmira, el litoral argentino-uruguayo y el proyecto de hidrovía del Paraguay. Finalmente, la sección de conclusiones presentará un balance de este problema, el estado actual de ambas iniciativas y sus potencialidades como casos de estudio para el análisis de la relación integración y desarrollo.

\section{LA RELACIÓN INTEGRACIÓN REGIONAL-DESARROLLO EN PERSPECTIVA HISTÓRICA: PROPUESTAS Y PROBLEMAS EN DEBATE}

En esta sección se presenta la discusión sobre la relación entre proyectos de integración y modelos de desarrollo con base en la literatura producida en la época, siguiendo un recorrido histórico para identificar las formas de articulación entre ambos procesos y los temas en discusión en el transcurso de un ciclo que se extiende desde la década de 1950 a la segunda década del siglo XXI.

Con la fundación de CEPAL en 1948, este centro de producción de ideas y propuestas fue dirigido por Raúl Prebisch y un equipo que integraba sociólogos y economistas. En ese marco, se origina la primera asociación de las categorías de desarrollo y espacio territorial integrado. En un texto publicado originalmente por El Trimestre Económico de México en 1983 y reimpreso en 1987, Raúl Prebisch diferencia cinco etapas en la evolución de su pensamiento sobre desarrollo y lo explica como respuesta a la insuficiencia de las teorías neoclásicas ante la crisis de la economía mundial de 1929 (Prebisch, 1987). El sistema centro-periferia proporcionaba en cambio una explicación de la desigualdad entre productores y exportadores de bienes primarios, caracterizada por la exagerada absorción del ingreso de la periferia por los países del centro, particularmente por los países industrializados favorecidos por el avance previo del progreso técnico (Bernal-Meza, 2005, pp. 78-94).

Las ideas de inaplicabilidad de las teorías económicas del centro en países de la periferia, del posible rol activo del Estado en el desarrollo y el papel del progreso 
técnico, establecieron el marco de las propuestas formuladas desde 1949, primero desde la CEPAL y luego desde la UNCTAD. Estas propuestas se dirigían a revertir los lineamientos del modelo de "crecimiento hacia afuera" con un nuevo patrón basado en la industrialización. Sin embargo, la complejidad de ese proceso exigía una aproximación que excedía la teoría económica y necesariamente requería incorporar la estructura social para explicar cómo en la periferia, el excedente económico era apropiado por los dueños de los medios de producción. Adicionalmente, la penetración y propagación de tecnología en los países de la periferia era más lenta de lo requerido para la absorción productiva de toda la fuerza de trabajo. La concentración del progreso técnico y todos sus frutos en actividades orientadas a las exportaciones habría consolidado una estructura social heterogénea en la cual gran parte de la población permanecía al margen del desarrollo.

Con base en esa crítica del modelo de crecimiento hacia afuera, Prebisch propuso un nuevo patrón de desarrollo basado en la industrialización para superar las limitaciones del modelo anterior. En su análisis de la quinta etapa de su pensamiento Prebisch (1987) anota que el problema central era encontrar una respuesta a la comprobación de que el desarrollo era acompañado de aumento en las disparidades sociales: una nueva revisión crítica de las ideas condujo a la conclusión de que los factores económicos no podían estudiarse sin analizar la estructura social.

Otros abordajes desde organismos de Naciones Unidas sobre desarrollo económico tuvieron por eje central el concepto de crecimiento: así, el texto del economista caribeño Arthur Lewis (1970) se concentra en las condiciones que hacen posible el crecimiento y las implicaciones para los países pequeños. Según Lewis, el desarrollo se produce en territorios cuyas instituciones y cultura estimulan el progreso y las transformaciones sociales: los procesos de desarrollo están condicionados por factores culturales como el espíritu de trabajo, la capacidad de ahorro y normas y reglas que regulan personas y organizan el territorio.

Lewis recomendaba a los países no desarrollados reducir sus importaciones desde los países desarrollados y en cambio fomentar el intercambio entre ellos: para ello era necesaria una red de relaciones comerciales y nuevas instituciones para financiarla. EI objetivo establecido para el Decenio del Desarrollo iniciado en 1960 era una tasa media de crecimiento del PBI del 5\% anual para la totalidad de los países menos desarrollados. Según Lewis, estaba claro que los países menos desarrollados podían 
crecer rápidamente siempre y cuando el comercio mundial estuviera en expansión (Lewis, 1970, pp. 12). Sus recomendaciones eran: aumentar la producción de alimentos y manufacturas livianas, y la creación de sistemas de preferencias comerciales mediante el establecimiento de uniones aduaneras, mercados comunes y zonas de libre comercio (Lewis, 1970, pp. 23).

La incidencia del regionalismo como medio efectivo para promover el desarrollo económico es el problema que Krauze (1961) aborda con un análisis de la aplicabilidad de una estrategia regional y de las fortalezas de los acuerdos regionales. Según el autor, algunos proyectos sólo son viables si tienen respaldo en bases regionales con la participación de dos o más países: sería ese el caso de las instalaciones hidroeléctricas, de regadío, o de transporte. La ausencia de complementación entre países no desarrollados es un obstáculo para el comercio recíproco entre ellos (Krauze, 1961, p. 515) pero la introducción de empresas de tipo industrial en países de una región sub-desarrollada puede ayudar a crear una mayor complementación entre esos países la cual, a su vez, haría posible un mayor intercambio dentro de la región. Según esta lógica, es posible "argüir en pro de acuerdos comerciales regionales con el fin de...promover el desarrollo económico".

En el análisis de las potencialidades del regionalismo como medio para impulsar el desarrollo, Krauze identifica los siguientes factores:

1) El desarrollo económico requiere mayor producción: esta a su vez requiere de nuevas empresas y algunas de ellas pueden crearse de forma más económica sobre bases regionales.

2) El marco regional es el que mejor cultiva el comercio entre países vecinos.

3) La implementación del intercambio regional en países subdesarrollados es obstaculizada por dos barreras principales: la falta de complementación y la falta de mecanismos de pago.

4) El crecimiento del comercio intra-regional depende de dos factores: la producción y un mecanismo monetario seguro.

Los casos del Mercado Común Centroamericano, fundado en 1951 y de la Asociación Latinoamericana de Libre Comercio (ALALC), creada en 1960, demostraban cómo la industrialización requería un mercado regional para un desarrollo en escala, pero también debía superar problemas de bajo acatamiento de los acuerdos y las políticas monetarias. La fundación del Mercado Común Centroamericano fue resultado de un 
rol activo de CEPAL (Calderón, 1968) y ejemplifica el modelo cepalino de desarrollo en su primera etapa: la experiencia de las industrias integradas con recursos de diferentes países del Istmo ilustra un modelo de regionalismo que avanzaba más allá de la sustitución de importaciones.

Francisco Thoumi (1985) presenta un balance del desarrollo cepalino en su primera época. Este trabajo destaca cómo los países menos desarrollados tienden a ser los perdedores en procesos de integración: sus economías son menos diversificadas que las de los más avanzados, el sector manufacturero está concentrado en actividades productivas que procesan recursos naturales; los mercados mayores que surgen de la integración tienden a favorecer industrias que no tienen competidores en otros países en términos de escala y que requieren mano de obra capacitada. Por consiguiente, las dinámicas de la integración operarían también contra los países menos desarrollados. Las expectativas en una distribución de costos y beneficios de la integración dieron origen al establecimiento de concesiones para los países menos desarrollados de los sistemas de integración latinoamericana: Thoumi destaca el tratamiento preferencial otorgado a Ecuador y Bolivia en el Mercado Común Andino, el de Paraguay en la Asociación Latinoamericana de Libre Comercio, y de Honduras en el Mercado Común Centroamericano. En su mayor parte esas concesiones habrían estado dirigidas a promover las exportaciones manufactureras de estos países a otros países miembros del bloque de integración.

Este estudio concluía que las diferencias en el grado de desarrollo entre países de una región pueden transformarse en poderosos obstáculos a la integración. En segundo lugar, los sistemas de integración económica podrían ofrecer mejores expectativas de éxito si incluyeran acuerdos bilaterales entre países que comparten regiones de frontera: el desarrollo de estas contribuiría a la integración regional.

En un análisis de los resultados de este modelo de desarrollo, los economistas Ffrench Davis, Muñoz y Palma (1997, p. 83) encuentran que la industrialización por sustitución de importaciones produjo algunos resultados positivos porque la economía se expandió rápidamente entre 1950 y 1981, el producto interno bruto creció con una tasa anual del $5,3 \%$, pero las desigualdades en la distribución del ingreso persistieron. Hubo un flujo importante de inversión extranjera directa concentrada en la producción sustitutiva de importaciones aunque a la vez surgieron nuevas formas de dependencia de la economía internacional, especialmente por la importación de insumos y 
tecnología. Sin embargo, desde otras perspectivas teóricas se anotaban las insuficiencias del modelo de desarrollo, en particular la persistencia de la dependencia como problema no resuelto por las estrategias de desarrollo económico (Cardoso y Faletto, 1987).

Un caso exitoso de aplicación de las recomendaciones de CEPAL fue el Mercado Común Centroamericano, fundado a partir de la creación en 1951 de un Comité de Cooperación Económica del Istmo Centroamericano y de la fundación, ese mismo año, de la ODECA (Organización de Estados Centroamericanos), el cual alcanzó un grado de integración importante de factores de producción en el sector industrial con la formación de empresas mixtas con capitales y mano de obra de los cuatro Estados miembros (López Cervantes, 2012).

Los orígenes de las propuestas de integración regional latinoamericana estuvieron asociados a estrategias de desarrollo que tenían como referencia las preguntas y debates del período post-gran depresión sobre tópicos como el modelo posible para lograr "un desarrollo extendido y sincrónico (mundial o regional)", la identificación del país "locomotora" que pudiera generar efectos de arrastre, o las expectativas generadas en el nivel macro-económico por el "empresario innovador" (Fuentes; Villanueva, 1989, p. 5).

En particular, ese empresario "shumpeteriano" creador de nuevas alternativas económicas y acompañado por el inversor- financista, según Fuentes y Villanueva, sería el encargado de extraer a la sociedad del estancamiento. Los autores anotan que en las décadas de 1950 y 1960 se lograron ciertas convergencias mundiales de conceptos y objetivos como el pleno empleo, crecimiento económico sostenido, ampliación general del mercado internacional de mercancías y un cierto consenso optimista respecto del desenlace de las tensiones existentes dentro de los países industrializados y en desarrollo. La sustitución de importaciones industriales, basada en inversión externa directa, era entendida como una estrategia de modernización. Este abordaje del desarrollo experimentó un vuelco al comienzo de la década de 1980 con el surgimiento de un nuevo modelo vinculado a la conformación de un sistema global e integrado por la acción determinante de las fuerzas transnacionales.

La transición en el regionalismo cepalino es la respuesta latinoamericana a ese cambio en la economía global. El documento de CEPAL (1994) proponía un nuevo 
modelo de integración regional que se apartaba de los lineamientos desarrollados en el período de la dirección de Prebisch: el regionalismo abierto.

Esta propuesta se basaba en una liberalización amplia de los mercados de bienes y servicios, normas estables y transparentes de acuerdo con las disposiciones del GATT, y fortalecimiento de las instituciones regionales que aportan financiamiento a la balanza de pagos a fin de minimizar posibles desequilibrios macroeconómicos. CEPAL (1994) recomendaba aplicar aranceles de protección moderada frente a terceros países y favorecer la aplicación de aranceles externos comunes en forma gradual si fuera necesario; armonizar arreglos institucionales, convertibilidad de monedas y elaboración de convenios de pagos; adoptar medidas especiales para favorecer el ajuste de los países y regiones con menor desarrollo relativo y a la vez brindar incentivos a la inversión intrarregional, construir infraestructura para minimizar los costos de transacción entre países y favorecer mecanismos institucionales flexibles.

De acuerdo con el análisis de Fuentes (1994) este cambio respondía a un nuevo contexto regional con la transición a la democracia de un grupo de países de América del Sur, la formación del Grupo de Río y la afirmación de la idea de la integración como instrumento que fortalece la democracia. Adicionalmente, la culminación de las negociaciones con la firma del Tratado de Libre Comercio de América del Norte, marcó la ruta en la formación de nuevos acuerdos regionales: la fundación de MERCOSUR en 1991 y la firma de varios acuerdos bilaterales por Chile ejemplifican esa tendencia.

En América Latina, la integración regional ingresó en una nueva etapa condicionada por nuevas estrategias de desarrollo orientadas hacia la adaptación a una economía mundial cada vez más exigente, procurando simultáneamente la competitividad de los países latinoamericanos. En la década de 1990 la integración se caracteriza por la proliferación de acuerdos de libre comercio mayoritariamente de formato bilateral, gran expansión del comercio y las inversiones recíprocas. Si bien la liberalización del comercio continuó siendo limitada, era mayor que en la fase anterior al igual que las reducciones arancelarias.

Los acuerdos comerciales incluían el sector de servicios, con la excepción del transporte marítimo y aéreo. Los planes de programación industrial a nivel subregional como los que fueran impulsados por el Mercado Común Centroamericano y el Pacto Andino, al igual que el interés por la formación de instituciones comunes, desaparecen del nuevo modelo de integración que Fuentes caracteriza como 
"superficial" en sustitución de la integración "profunda." En cambio, se observa un proceso de racionalización de las actividades de las filiales de empresas transnacionales que progresivamente adoptan metas regionales o sub-regionales (Fuentes, 1994, p. 83).

Con el cambio de milenio, el impacto de la crisis económica-financiera entre 1998 y 2002 en el Cono Sur, y la transición política en el gobierno en varios países latinoamericanos, nuevas propuestas de integración regional recuperan algunos objetivos de desarrollo de los proyectos integracionistas del período anterior a las políticas de internacionalización de las economías latinoamericanas. Iniciativas como los avances en la Unión Aduanera de MERCOSUR tras la aprobación del Código Aduanero en 2010, el Protocolo de Comercio de Servicios aprobado en 2003, las propuestas de integración productiva y de tratamiento de las asimetrías entre estados miembros del bloque son, según Quijano (2011), evidencia de un giro hacia el regreso a la integración profunda. La literatura sobre regionalismos, Sanahuja (2009), Riggirozzi y Tussie (2012), Briceño (2006), caracteriza el viraje en el modelo de integración como post-liberal, post-hegemónico, o estratégico, marcado por el regreso del Estado y del desarrollo como prioridad en la agenda.

Sin embargo, el análisis de las relaciones intra-bloque revela en el caso de MERCOSUR la existencia de desconfianza y conflictos entre socios y plantea nuevos problemas a la investigación en torno a la solidez de la construcción políticoinstitucional de la integración. Por otra parte, la importancia creciente de las relaciones con las potencias emergentes del Asia, entre las cuales China pasó rápidamente a la condición de gran potencia, produjo un crecimiento sostenido de las exportaciones latinoamericanas, con un fuerte componente de bienes primarios, hacia los mercados asiáticos. En un contexto de optimismo originado en los buenos resultados del comercio exterior, surgió la discusión sobre los alcances de la relación integración y desarrollo, en el marco más amplio del debate sobre los modelos de desarrollo.

\section{Alternativas del desarRollo endógeno, local y/o sostrenible. DesarRollo} LOCAL, INTEGRACIÓN Y TERRITORIO, MEDIO AMBIENTE E INTEGRACIÓN

Los insuficientes resultados en términos de logros de desarrollo y la conciencia cada vez más extendida acerca de los riesgos de ruptura en los equilibrios entre sociedad humana y medio ambiente natural, condujeron a una revisión del modelo de desarrollo 
y a la formulación de nuevas propuestas en las cuales la dimensión territorial adquirió relevancia.

El modelo de desarrollo endógeno incluye enfoques diversos que adoptan una aproximación territorial. Según Vázquez Barquero (2007, p. 183) este modelo incluye conceptos elaborados a partir de la década de 1980 desde otros enfoques como desarrollo auto-centrado, desarrollo sostenible, desarrollo humano y desarrollo desde abajo. Integra el crecimiento de la producción social e instituciones del territorio a la política de desarrollo territorial que exige un rol protagónico de la sociedad civil en la planeación y ejecución de la economía.

El punto de partida de esta aproximación es una evaluación crítica desde la ciencia social sobre el pensamiento económico dominante en la academia y de la interpretación que desde la II Guerra Mundial influyó en las investigaciones y en los lineamientos de acción de los organismos internacionales con mayor incidencia en políticas de desarrollo. En América Latina, la influencia del modelo de desarrollo endógeno se manifestó principalmente en Venezuela en la primera década del milenio y posteriormente en el bloque sub-regional ALBA-TCP.

De acuerdo con Parker (2017) a partir de 2003, el gobierno venezolano adoptó el concepto de desarrollo endógeno, el cual ya estaba en circulación entre economistas de países desarrollados, principalmente en referencia a procesos de crecimiento económico local o regional y a sinergias y generación de capital social con incorporación de avances tecnológicos para competir en el mercado globalizado. En la explicación de Vázquez Barquero (2001) no hay cuestionamientos ni críticas al neoliberalismo. Pero es sobre todo la obra de Osvaldo Sunkel (1991) la influencia mayor en la concepción del proyecto de desarrollo endógeno en Venezuela.

Muy mencionado en el debate político venezolano y en los textos escritos sobre desarrollo y política económica en la época, la obra de Sunkel dio lugar a lecturas muy diversas por su carácter de obra colectiva. Si el análisis se concentra en el capítulo introductorio del libro, escrito por Sunkel, Vera y Ramos, allí se encuentra que afirman que su abordaje del desarrollo significa retomar y superar el desafío industrializador propuesto por Prebisch para generar un mecanismo endógeno de acumulación y generación de progreso técnico que permita desarrollar capacidades propias para crecer con dinamismo y productividad. Retomando la idea de Sunkel en cuanto a que la prioridad debe ser pagar la deuda social a través de una reactivación selectiva de la 
economía, el objetivo principal en el corto plazo sería el combate a la pobreza y la elaboración de políticas de largo plazo con el fin de superar la pobreza extrema mediante cambios distributivos dirigidos a lograr un nivel de equidad socialmente aceptable y promover el progreso técnico. Entre esas medidas, se destacan el apoyo a pequeñas y medianas empresas y a los sectores informales.

En 2003, Chavez anuncia su adhesión al modelo de desarrollo endógeno. Sin embargo, la interpretación del concepto de desarrollo endógeno expuesto en Sunkel es en Venezuela muy flexible y en varios departamentos del gobierno se mantuvo el modelo cepalino: Parker cita un documento de abril de 2003, del Ministerio de la Producción y el Comercio, que propone un Plan de desarrollo endógeno de la industria venezolana que prevé una política industrial para la sustitución de importaciones. Otras entidades del estado asocian este modelo con el desarrollo social y el papel de las misiones sociales. Elías Jauá (2005) sintetiza el pensamiento del gobierno que da sustento a las políticas nacionales vinculadas a la política de integración.

La fundación de ALBA en 2001 se hizo efectiva en 2004 con una composición que sumaba países sudamericanos y caribeños. Romero (2009) sostiene que el propósito inicial fue impulsar una nueva articulación de desarrollo e integración. Shaposnik y Pardo (2014) afirman que los objetivos de desarrollo en este bloque regional tienen como prioridades el sector energético, con la creación de Petrocaribe, el comercio mediante convenios de intercambio compensado, la producción industrial enmarcada en las empresas gran-nacionales y el desarrollo e integración social con base en organizaciones como el Consejo Social, el Comité de la Mujer y la Igualdad y el Consejo de Movimientos Sociales.

Contemporáneamente, en América Latina, el enfoque territorial parte del reconocimiento de la complejidad y diversidad de visiones sobre desarrollo e involucra diversos planos: productivo, tecnológico, social, cultural y político. Ideas centrales en este enfoque son el énfasis en los procesos de acumulación y crecimiento en los territorios. El modelo de desarrollo local expuesto por Arocena (2002) asigna un papel determinante a la iniciativa local que emerge de la sociedad y a los procesos de descentralización y la consiguiente formación de instituciones de gobierno local como actores potenciales del desarrollo. Pero el desarrollo local no se confunde con gobierno local: según Arocena, la especificidad del desarrollo local reside en el origen 
local de las iniciativas y los recursos, el involucramiento de la sociedad local en ellas, y las dinámicas de transformación socio-económica que se producen en el territorio.

Con el advenimiento del tercer milenio, nuevas visiones de desarrollo inciden en algunos organismos multilaterales y por ese medio, ingresan en la discusión latinoamericana: la obra de Amartya Sen, adoptada en el PNUD proporciona la base del modelo de desarrollo humano y la asociación de factores de desarrollo, creatividad y capacidad. Según Amartya Sen (2000), desarrollo es más que crecimiento y es esencial la conexión desarrollo-libertad.

Al mismo tiempo, la discusión sobre modelos de desarrollo en el marco de procesos de integración introduce nuevos temas en los acuerdos regionales. El concepto de desarrollo sostenible emerge tempranamente en MERCOSUR, según Amalia Stuhldreher (2012): el tratado de Asunción incluía la meta de desarrollo económico con justicia social, un objetivo que debía ser alcanzado con el uso eficaz de los recursos naturales, la preservación del medio ambiente y la coordinación de políticas macroeconómicas. La declaración de Canela (1992) intentó fijar una posición común sobre temas ambientales y un compromiso con el desarrollo sostenible mediante el uso racional de los recursos naturales. Entre los objetivos propuestos, se destaca la cooperación científica y tecnológica con países desarrollados. A pesar de esos avances, la autora destaca que no hubo consenso para acordar un protocolo adicional al tratado de Asunción sobre medio ambiente. Finalmente, en 2001 se produjo la aprobación del Acuerdo Marco sobre Medio Ambiente.

\section{ANÁLISIS DE DOS ÁREAS DE MERCOSUR Y MODELOS DE DESARROLLO}

En esta sección se presentan dos casos diferentes que incluyen proyectos de clara vinculación con problemas locales de desarrollo económico y social, involucran participación de actores locales, experiencias de desarrollo endógeno y acción coordinada entre entidades de gobierno nacional y sub-nacional. En ambos se observa el efecto de la integración sub-regional y sudamericana. Especialmente el proyecto de integración y modernización de la infraestructura física, de transportes, energía y comunicaciones de América del Sur, sustentado en la participación de instituciones financieras creadas en el marco regional, BID, CAF y FONPLATA, produjo cambios en la significación de los territorios en los dos casos que se presentan a continuación. 


\section{Integración, cooperación transfronteriza y desarrollo en la frontera} uruguayo-brasileña. Si bien los proyectos que se analizan en este apartado se originan en acuerdos de alcance bilateral, se inscriben en el marco mayor del bloque sub-regional y se apoyan en instrumentos generados por la integración, como los Fondos de Convergencia Estructural del Mercosur (FOCEM). Por otra parte, La Nueva Agenda de Cooperación y Desarrollo Fronterizo aprobada por los gobiernos de Uruguay y Brasil en 2002 comprende proyectos de diversa naturaleza: permiso de residencia, estudio y trabajo para los habitantes del territorio delimitado como "faja de frontera", saneamiento de los municipios Aceguá (Brasil) y Aceguá (Uruguay), restauración del puente sobre el río Yaguarón y construcción de un segundo puente, hidrovía formada por lagunas Merín y de Los Patos, y ríos afluentes y proyectos de interconexión eléctrica a cargo de las empresas estatales de los dos países (Clemente y Mallmann, 2016, pp. 95-108). Aun cuando el nivel de ejecución de estos proyectos ha sido desigual, ilustra la diversidad de temas de la agenda del desarrollo, en superación de la concentración casi exclusiva en la promoción del comercio que identifica la economía de la integración desde la década de los años 1990.

La articulación de algunos de estos objetivos con la planeación de la infraestructura sudamericana está presente en los casos de los proyectos de adecuación del corredor Río Branco-Montevideo-Colonia-Nueva Palmira, y de construcción de un nuevo puente internacional Jaguarão-Río Branco.

\section{Articulación territorio, integración y desarrollo en el eje de la hidrovía}

\section{Paraguay.}

El caso del puerto uruguayo de Nueva Palmira, situado en las nacientes del Río de la Plata, ejemplifica las modalidades del desarrollo local y su vinculación con movimientos transnacionales: la remodelación del puerto, finalizada en 1990, marcó el comienzo de una transformación y crecimiento en la actividad portuaria. Ello se refleja en las cifras de empleo (Magri, Altair; Abrahan, Manuela; Ogues, Leticia, 2015: 96101). Según los autores citados, la historia de Nueva Palmira aparece asociada desde la época colonial a un puerto que se inicia con un muelle fluvial sobre el río de la Plata. La navegación hacia Salto por el curso del río Uruguay fue seguida de un incipiente desarrollo local impulsado por la industria aceitera y de armado de automóviles. Desde la construcción de un puerto de ultramar en 1931, a la ampliación y mejoras en 1985, a la entrada en vigencia de la hidrovía del Paraguay se procesa una transformación del 
puerto y su conexión con exportaciones de granos (principalmente soya) y celulosa. (Abrahan, Beder, Cánepa y Goinheix, 2014, pp. 96-101). El desarrollo local revela los efectos de la transición de un sistema productivo centrado en la industria a un modelo exportador. Los autores identifican cierta precariedad en el empleo, rezago en la educación y un papel importante de la inmigración.

Según Adrián Rodríguez y Carlos Troncoso (2015) la ubicación estratégica de Nueva Palmira como puerto de salida de la hidrovía Paraná-Paraguay y el boom agrícola dirigido a la exportación de granos explican la transformación de este puerto en un complejo agro-portuario que genera dinámicas locales de producción en el territorio vecino (Rodríguez Miranda, Adrián; Troncoso, Carlos, 2015: pp. 124-128). La internacionalización de Nueva Palmira, otrora un pacífico puerto local, responde no sólo a las lógicas transnacionales sino también a la integración y a las necesidades de conexión externa de dos países mediterráneos de América del Sur: Paraguay, el principal beneficiario y en menor medida, Bolivia.

\section{IV.CONCLUSIONES}

En el período analizado en este trabajo, la asociación integración regional-desarrollo económico de América Latina se mantuvo constante y en términos de objetivos, la integración fue entendida como la vía para cumplir planes de desarrollo económico de la región. Sin embargo, si la vocación integracionista se mantuvo constante, hubo variaciones importantes en cuanto al modelo de integración y en cuanto al modelo de desarrollo.

Estas variaciones tuvieron como tema central el rol del Estado: en la primera fase, caracterizada por la integración asociada al modelo económico de industrialización por sustitución de importaciones, la intervención del Estado fue activa y los sistemas de integración tuvieron un grado importante de institucionalización en apoyo al proceso integracionista.

El viraje hacia el regionalismo abierto dio paso a la reducción del papel del Estado en la integración regional, en coherencia con la transferencia al mercado de un conjunto importante de funciones antes desempeñadas por el Estado en el marco de las políticas públicas. En ese sentido, el nuevo modelo de integración adoptado en la década de 1990 acompaña el movimiento general hacia la desregulación. 
La transición hacia el neo-estatismo desde inicios del tercer milenio marcó un retorno del Estado en áreas de políticas de integración vinculadas a desarrollo social y desarrollo productivo, infraestructura y logística, sin cambios sustantivos en las políticas de comercio exterior. Simultáneamente, la acción independiente de las organizaciones de la sociedad civil generó nuevas dinámicas en el nivel local y diversificación de agendas de desarrollo.

$\mathrm{Si}$ bien la relación integración regional-desarrollo se mantuvo constante, la participación de los actores locales se fortaleció y los problemas centrales en el modelo de desarrollo giraron desde la inserción latinoamericana en el comercio internacional a la defensa de los equilibrios entre crecimiento económico, desarrollo social y sustentabilidad ambiental en los territorios.

\section{BiBLIOGRAFÍA}

Abrahan, M., Beder, F. Cánepa, G. \& Goinheix, S. (2015). Capitulo 1. La Paloma y Nueva Palmira: trayectoria histórica y datos socio-económicos actuales (pp. 95-110). En A. Magri, M. Abrahan \& L. Ogues. (Coords.), Nuevos desafíos y respuestas de los actores sobre el desarrollo local: La Paloma y Nueva Palmira frente a propuestas de inversión. Universidad de la República, Montevideo, Uruguay: Espacio Interdisciplinario. Recuperado de https://www.colibri.udelar.edu.uy/jspui/bitstream/123456789/7587/1/nuevos desaf\%C3 \%ADos y respuestas.pdf

Arocena, J. (2002). El desarrollo local: un desafío contemporáneo. Montevideo, Uruguay: Taurus-Universidad Católica del Uruguay. Recuperado de http://biblioteca.municipios.unq.edu.ar/modules/mislibros/archivos/Arocena.pdf

Bernal-Meza, R. (2005). América Latina en el mundo. El pensamiento latinoamericano y la teoría de las relaciones internacionales. Buenos Aires, Argentina: NuevoHacer.

Briceño, J. (2009). El ALBA y el MERCOSUR en la agenda de integración de Venezuela: ¿Son compatibles?. En Briceño, J. \& Mendoza, C. (Eds.), Cambio y permanencia en la agenda de integración de América del Sur (pp.185-218). Barquisimeto, Venezuela: Fondo Editorial UCLA.

Briceño, J. (2006). Regionalismo estratégico e interregionalismo en las relaciones externas del Mercosur. Aportes para la integración latinoamericana, 12(15), 30-45. 
Calderón Martínez, A. (1968). El Mercado Común Centroamericano y la Asociación Latinoamericana de Libre Comercio. Revista de Comercio Exterior, 975-984. Recuperado de: www.revistas.bancomext.gob.mx/rce/magazines/744/5/RCES.pdf

Cardoso, F. \& Faletto, E. (1987). Dependencia y Desarrollo en América Latina. Ensayo de interpretación sociológica. México: Siglo XXI Editores.

Clemente, I. \& Mallmann, M. I. (2017). Mercosur e integración fronteriza: la cooperación transfronteriza entre Brasil y Uruguay. En E. Mazzei (Ed.), Las sociedades fronterizas: visiones y reflexiones (pp.95-108). Montevideo, Uruguay: Universidad de la República-Comisión Coordinadora de Interior y Centro de estudios de la Frontera. Recuperado de http://desarrolloterritorial.ei.udelar.edu.uy/wpcontent/uploads/2017/05/Sociedades-fronterizas-WEB.pdf

Comisión Económica para América Latina (CEPAL). (1994). El regionalismo abierto en América Latina y el Caribe. La integración económica al servicio de la transformación productiva con equidad. Santiago de Chile. Recuperado de https://repositorio.cepal.org/handle/11362/2140

De Lombaerde, P. \& Garay, L. J. (2006). The New Regionalism in Latin America and the role of the US. Occasional Papers, UNU-CRIS. Recuperado de http://cris.unu.edu/sites/cris.unu.edu/files/O-2006-10.pdf

French-Davis, R., Muñoz, O. \& Palma, J. G. (1997). Las economías latinoamericanas, 1950-1990. En L. Bethell. (Ed.), Historia de América Latina. 11 Economía y Sociedad desde 1930 (vol. 11). (pp. 83-162). Barcelona: Cambridge University Pres-CríticaGrijalbo-Mondadori..

Fuentes, A. \& Villanueva, J. (1989). Economía mundial e integración de América Latina. Buenos Aires, Argentina: INTAL-BID.

Fuentes, J. A. (1994). El regionalismo abierto y la integración económica. Revista CEPAL, (53), 81-90. Recuperado de https://www.cepal.org/es/publicaciones/11951regionalismo-abierto-la-integracion-economica

Ibrahim, S. \& Alkire, S. (29 Nov. 2007). Agency and Empowerment: A Proposal for Internationally Comparable Indicators. Oxford Development Studies, 35(4), 379-403. Recuperado de https://papers.ssrn.com/sol3/papers.cfm?abstract id=2118589 
Ponente, Jauá, E. (Junio, 2005). Nuevo modelo socio-productivo y desarrollo endógeno. Primer Simposio Nacional sobre Planificación y Desarrollo Endógeno Caracas, Venezuela: MINEP.

Krause, W. (1961). El desarrollo económico a través del regionalismo. Journal of Interamerican Studies, 3(4), 509-525.

Lewis, W. A. (1970). El proceso de desarrollo. Nueva York, EEUU: Naciones Unidas.

López Cervantes, G. (2012). El Mercado Común Centroamericano. Mediterráneo Económico, 249-269.

Recuperado de

http://www.publicacionescajamar.es/publicaciones-periodicas/mediterraneoeconomico/mediterraneo-economico-22-la-economia-internacional-en-el-siglo-xxi/

Lugo, M. A. (2007). Employment: A Proposal for Internationally Comparable Indicators. Oxford Development Studies, 35, (4), 361-378.

Magri, A., Abrahan, Mazzolenni, M. \& Ogues, L. (Coords.). (2015). Nuevos desafíos y respuestas de los actores sobre el desarrollo local. La Paloma y Nueva Palmira frente a propuestas de inversión. Montevideo, Uruguay: Universidad de la República-Espacio Interdisciplinario.

https://www.colibri.udelar.edu.uy/jspui/handle/123456789/7587b

MERCOSUR/CMC. Dec. 2/01 Acuerdo Marco sobre Medio Ambiente (2001). Disponible en: www.mercosur.int/innovaportal/file/176/1/st - 3 pdf

Parker, D. (2017). El desarrollo endógeno ¿Camino al socialismo del Siglo XXI? Revista Venezolana de Economía y Ciencias Sociales, 13(32). Recuperado de https://www.redalyc.org/articulo.oa?id=17721547005

Prebisch, R. (1987). Cinco etapas de mi pensamiento sobre el desarrollo. Comercio Exterior. 27(5), 345-352.

Puglia Mascaronis, A. (2011). Los fondos de convergencia estructural del Mercosur. Un enfoque distinto del proceso de integración. En M. Cienfuegos, N. Mellado \& W. N. Fernandez. (Eds.), Los cambios en la infraestructura regional y sus impactos ambientales en clave de mejorar la gobernabilidad en el Mercosur (pp. 175-182). Córdoba, Argentina: Lerner Editora. Recuperado de http://sedici.unlp.edu.ar/handle/10915/59805 
Quijano, J. M. (2011). El MERCOSUR 20 años después. En G. Caetano. (Coord.), MERCOSUR: 20 años (pp. 89-133). Montevideo, Uruguay: CEFIR, Friedrich Ebert Stiftung, GIZ, Somos Mercosur.

Riggirozzi, P, \& Tussie, D. (2012). (Eds.). The Rise of Post-Hegemonic Regionalism. The Case of Latin America. Londres, UK: Springer.

Rodríguez Miranda, A. \& Troncoso, C. (2015). Sistemas productivos locales ¿proyectos empresariales o territoriales? Los casos de Nueva Palmira y La Paloma. En A. Magri, M. Abrahan \& L. Ogues. (Eds.), Nuevos desafíos y respuestas de los actores sobre el desarrollo local. La Paloma y Nueva Palmira frente a propuestas de inversión (pp. 111-140). Montevideo, Uruguay: Universidad de la República-Espacio Interdisciplianrio.

https://www.colibri.udelar.edu.uy/jspui/handle/123456789/7587

Romero, N. (2009). El ALBA ¿Una vía hacia un nuevo modelo de desarrollo e integración?. En J. Briceño, \& C. Mendoza. (Eds.), Cambio y permanencia en la Agenda de la Integración de América del Sur (pp. 161-184). Barquisimeto, Venezuela: Fondo Editorial UCLA.

Sanahuja, J. A. (2009). Del Regionalismo abierto al Regionalismo Post-Liberal. Crisis y Cambio en la integración de América Latina y el Caribe 2008-2009. Anuario de la Integración de América Latina y el Gran Caribe, CRIES, (7), 11-54. Recuperado de https://eprints.ucm.es/42566/

Secretaría MERCOSUR. (2006). Medio Ambiente en el MERCOSUR. Relevamiento $N^{\circ}$ 001/06. Recuperado de http://docplayer.es/14586208-Medio-ambiente-en-el-mercosurrelevamiento-no-001-06.html

Sen, A. (2000). El desarrollo como libertad. Gaceta Ecológica, (55), 14-20. Recuperado de https://www.redalyc.org/articulo.oa?id=53905501

Schaponsnik, C. R. \& Pardo, E. C. (2014). La agenda social en el ALBA-TCP. En N. Mellado. (Ed.), Regionalismo Latinoamericano: dimensiones actuales (pp. 229-262). Córdoba, Argentina: Editora Lerner. Recuperado de http://sedici.unlp.edu.ar/handle/10915/59819

Stuhldreher, A. (2017). Cambio climático y desarrollo en regiones rezagadas de América Latina. Trabajo presentado en $9^{\circ}$ Congreso ALACIP. Montevideo, Uruguay. 
Stuhldreher, A. (2012). Construcción participativa del regionalismo estratégico: ¿hacia una agenda medioambiental externa del Mercosur?. Revista Brasileira de Política Internacional, 55(1), 194-210. Recuperado de http://www.scielo.br/scielo.php?pid=S0034$\underline{73292012000100011 \& \text { script=sci abstract\&tlng }=e s}$

Sunkel, O. (Ed.). (1991). El desarrollo desde adentro: un enfoque neo-estructuralista para América Latina. México: Fondo de Cultura Económica.

Thoumi, F. (1985). Intraregional Trade of the Least Developed Members of Latin American Integration Systems. Journal of Interamerican Studies and World Affairs, 27 (4), 75-94.

Vázquez Barquero, A. (2007). Desarrollo endógeno. Teoría y Políticas de desarrollo territorial. Investigaciones Regionales, (11), 183-210. Recuperado de https://www.redalyc.org/articulo.oa?id=28901109

Clemente Batalla ISABel: Doctora de la Universidad de Londres, Magister en Ciencia Política de la Universidad de los Andes, Bogotá. Profesora Agregada en el Programa de Estudios Internacionales de la Facultad de Ciencias Sociales, Universidad de la República. Docente de Negociación Internacional en el postgrado en Estudios Internacionales y de los cursos Sistema Internacional y Gobernanza Global en la Licenciatura en Desarrollo de la Facultad de Ciencias Sociales. Co-coordinadora con la Profesora María Izabel Mallmann de la Pontificia Universidad Católica de Río Grande do Sul del proyecto de investigación "Impacto da Agenda Binacional de Cooperação e Desenvolvimiento Fronteiriço sobre as relações entre Brasil e Uruguai", Programa CAPES-UDELAR, proceso selectivo 2011. Entre sus publicaciones recientes se encuentran: "Cambio y continuidad en la relación binacional Brasil-Uruguay," en: Malmann, María Izabel, y Scheider, Theresa Cristina (Organizadoras) Fronteiras e Relações Brasil Uruguai, Porto Alegre: EDIPUCRS, 2015, pp. 71-84. "Mercocidades e Integração regional" (en coautoría con María Izabel Mallmann), en Gomes, Joseli Fiorin y Corradi, Rodrigo de Souza (Orgs.) Paradiplomacia em movimiento, Porto Alegre: UniRitter, 2015, pp. 123-136. "De la 'razón de Estado' a la utopía: aproximaciones a El Príncipe de Maquiavelo", en: Instituto de Historia de las Ideas, Maquiavelo Intemporal en los 500 años de El Príncipe, Montevideo: FCUFacultad de Derecho, 2015, pp. 197-208. E-mail: Isabel.clemente@cienciassociales.edu.uy 